\title{
Correction to: Recognizing obsessive- compulsive disorder: how suitable is the German Zohar-Fineberg obsessive- compulsive screen?
}

Franziska Kühne*, Tatjana Paunov and Florian Weck

Following the publication of the original article [1], the authors identified that the funding note was incorrect. The correct funding note is given below.

Funding

This research did not receive any specific grant from funding agencies in the public, commercial, or notfor-profit sectors. We acknowledge the support of the Deutsche Forschungsgemeinschaft and Open Access Publishing Fund of University of Potsdam".

The original article [1] has been corrected.

Published online: 21 January 2022

\footnotetext{
Reference

1. Kühne F, et al. Recognizing obsessive-compulsive disorder: how suitable is the German Zohar-Fineberg obsessive-compulsive screen? BMC Psychiatry. 2021;21:450. https://doi.org/10.1186/s12888-021-03458-x.
} 